\title{
Photoinduced Dehydrogenation of Defects in Undoped $a$-Si:H Using Positron Annihilation Spectroscopy
}

\author{
X. Zou, Y. C. Chan, D. P. Webb, and Y.W. Lam \\ Department of Electronic Engineering, City University of Hong Kong, Tat Chee Avenue, Kowloon, Hong Kong, China \\ Y.F. Hu, C.D. Beling, and S. Fung \\ Department of Physics, The University of Hong Kong, Pokfulam Road, Hong Kong, China \\ H. M. Weng \\ Department of Modern Physics, University of Science and Technology of China, Hefei 230026, People's Republic of China
}

(Received 29 June 1998)

\begin{abstract}
We report changes in variable-energy positron annihilation spectroscopy measurements on undoped hydrogenated amorphous silicon films after light soaking. The change, seen predominantly in the high momentum band of the annihilation radiation, is not reversed by thermal annealing. We suggest, following recent models of the Staebler-Wronski effect, that light exposure induces hydrogen trapped in vacancylike defects to become mobile in the Si network. The observations place constraints on models of hydrogen motion fitting macroscopic Staebler-Wronski effect kinetics and may help to achieve a definitive description of metastability in $a-\mathrm{Si}: \mathrm{H}$.
\end{abstract}

PACS numbers: $78.70 . \mathrm{Bj}, 81.05 . \mathrm{Gc}$

Advances in amorphous hydrogenated silicon $(a-\mathrm{Si}: \mathrm{H})$ technology have been hindered by the limited understanding of the microstructure. In particular, shortly after the realization of effective doping of $a$-Si:H [1], it was found the material suffers from light-induced metastable changes [Staebler-Wronski effect (SWE)] [2]. Light incident on $a$-Si:H causes metastable Si dangling bond (DB) defects to form with the result of increased carrier trapping and decreased photoconductivity. Much theoretical and experimental effort has been put into understanding the SWE, but a comprehensive explanation has yet to be achieved.

Hydrogen is known to play a dominant role in the stabilization and annealing properties of the metastable defects [3]. A recent class of models [4-6], which explains much of the experimental data, has light-induced electron-hole pairs breaking $\mathrm{Si}-\mathrm{H}$ bonds and allowing $\mathrm{H}$ to become incorporated in a mobile form within the $a$-Si network. According to Biswas and Pan [4] and Branz [5], the light-induced $\mathrm{Si}^{*} \mathrm{DBs}$ are induced at the original site of hydrogen emission:

$$
\mathrm{Si}-\mathrm{H} \stackrel{\left(e^{-}-h^{+}\right)}{\longrightarrow} \mathrm{Si}^{*}+\mathrm{H}_{\text {mobile }} \text {. }
$$

The mobile form of $\mathrm{H}$ arises as a result of the atom jumping from one bond-center site to another in the form of $\mathrm{Si}-\mathrm{H} / \mathrm{Si}^{*}$ complex $[7,8]$ which reduces the energy cost of breaking the Si-H bond. In contrast, Godet [6] addresses the energy problem by suggesting hydrogen is emitted from doubly hydrogenated Si-HH-Si sites where reconstruction is permitted:

$$
(\mathrm{Si}-\mathrm{HH}-\mathrm{Si}) \stackrel{\left(e^{-}-h^{+}\right)}{\longrightarrow} \mathrm{Si}-\mathrm{Si}+2 \mathrm{H}_{\text {mobile }} .
$$

Here the mobile form of $\mathrm{H}$ is a three-center Si-H-Si configuration. The $\mathrm{DBs}$ result when the mobile $\mathrm{H}$ reacts with $\mathrm{Si}-\mathrm{H}$ bonds [6]:

$$
\mathrm{H}_{\text {mobile }}+\mathrm{Si}-\mathrm{H} \rightarrow \mathrm{Si}^{*}+(\mathrm{Si}-\mathrm{HH}-\mathrm{Si}) .
$$

Both models agree that it is the "sinking" of $\mathrm{H}$ atoms into low energy ( $\mathrm{Si}-\mathrm{HH}-\mathrm{Si}$ ) configurations that leads to the metastability, because this complex is stable at room temperature. It is only the mechanism of $(\mathrm{Si}-\mathrm{HH}-\mathrm{Si})$ production that differs. The scheme of Godet [6] favors reaction (2) while in the scheme of Branz [5] the (Si-HH-Si) complex forms on the collision of two mobile $\mathrm{H}$ atoms.

Both schemes can explain the observed kinetic data on DB formation and annealing. Evidence of hydrogen uptake comes from the observation of expansion of the $\mathrm{Si}$ network occurring concurrently with illumination [9-15] since on forming the mobile $\mathrm{H}$ state at the bond-center site there is an average $0.4 \AA$ outward relaxation in the $\mathrm{Si}-\mathrm{Si}$ separation [7]. However, to distinguish between the models requires direct information on the microscopic origin of such macroscopic changes. Positron annihilation spectroscopy (PAS) is a powerful tool for studying point defects in semiconductors since positrons naturally trap into open volume sites and through the annihilation radiation they emit microscopic information is made available [16]. Here we report on light soaking induced microstructural changes in the defect structure of undoped $a$-Si:H observed by PAS.

The two samples studied in this work, both giving similar results, were $1 \mu \mathrm{m}$ intrinsic $a$-Si:H homogeneous films grown by pure silane $\left(\mathrm{SiH}_{4}\right)$ PECVD (plasma enhanced chemical vapor deposition) at a deposition rate of $1.5 \mathrm{~nm} / \mathrm{s}$ [17] on quartz, at rf power $40 \mathrm{~W}$, and substrate temperature 
$330{ }^{\circ} \mathrm{C}$. The ratio of photoconductivity to dark conductivity of the film was about 5 orders, indicating material of solar cell quality. The light soaking illumination intensity was $100 \mathrm{~mW} \mathrm{~cm}^{-2}$. The Staebler-Wronski effect was observed in the electrical measurements.

The PAS measurements were performed using the monoenergetic positron beam at the University of Hong Kong, detailed in [18]. The maximum beam energy of $25 \mathrm{keV}$ gives a mean positron stopping depth of about $3 \mu \mathrm{m} \mathrm{[19]} \mathrm{in} a$-Si:H of density $2.2 \mathrm{~g} \mathrm{~cm}^{-3}$. The $511 \mathrm{keV}$ annihilation $\gamma$ radiation from the sample was detected with a high purity Ge detector of resolution 1.4 at $514 \mathrm{keV}$ and a digitally stabilized multichannel analyzer system. A total of $1 \times 10^{6}$ counts were collected under the annihilation $\gamma$-ray photopeak for each positron energy. The $\gamma$-ray photopeak line shape was described using the conventional line shape parameters $S$ and $W$, which are, respectively, the ratios of counts in the central and wing portions of the annihilation photopeak to the total peak count [20]. The $S$ parameter window was set to cover positron annihilations with low momentum valence electrons having a longitudinal momentum component of $p_{L} \leq 3.7 \times 10^{-3} m_{0} c$, where $m_{0}$ is the electron mass and $c$ is the speed of light, and the $W$ parameter is to cover high momentum core electrons over the range $7 \times 10^{-3} m_{0} c \leq p_{L} \leq 23 \times 10^{-3} m_{0} c$.

Positrons get trapped at neutral and negative vacancy defects because the missing positive charge of the ion cores causes the particle to experience a potential well. The reduced valence and core electron density at a vacancy increases the lifetime of the trapped positron, and narrows the positron momentum distribution, since the relative proportion of valence to core annihilation increases. Consequently, with an increasing number of microvoids, $S$ increases and $W$ decreases. The ratio of $S$ to the bulk crystalline value $S_{\text {bulk }}$ is an approximate gauge of the type of defect. For example $S / S_{\text {bulk }}$ for monovacancies is 1.030 , divacancies 1.034-1.038, vacancy clusters 1.047-1.061, and microvoids 1.10-1.14 [20,21]. Figure 1(a) shows the variation of $S / S_{\text {bulk }}$ vs incident energy for as-grown, lightsoaked, and $40-\mathrm{h} 150{ }^{\circ} \mathrm{C}$ annealed $a-\mathrm{Si}: \mathrm{H}$ thin films. The same general trends are observed in all samples, namely, a plateau in the $2-10 \mathrm{keV}$ range characterizing the $a-\mathrm{Si}: \mathrm{H}$ film, while $S$ drops at lower and higher energies due to the surface and substrate, respectively. The ratios $S / S_{\text {bulk }}$ for the as-deposited, light-soaked, and annealed films in the plateau regions are 1.018, 1.021, and 1.018, respectively, indicating annihilation both from the bulk and probably small size trapping vacancies. The $S_{\text {bulk }}$ value was measured separately on a crystalline $\mathrm{Si}(c-\mathrm{Si})$ sample with our equipment.

The results show little process condition dependency in the $S$ parameter of the film, agreeing with Muramatsu et al. who also noted no appreciable change in $S$ on light soaking [22]. In sharp contrast is the very noticeable change in the $W$ parameter on light soaking shown in
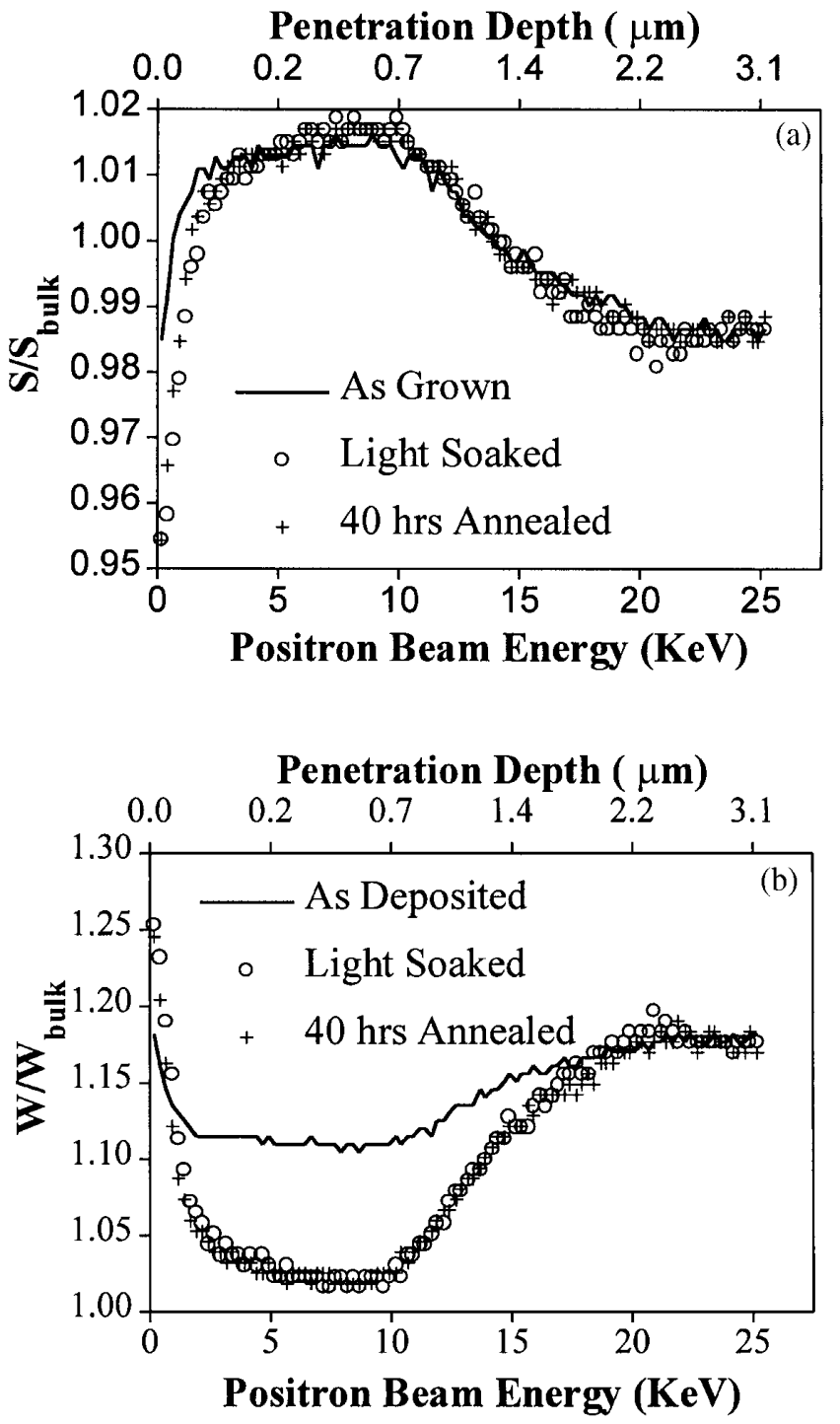

FIG. 1. The (a) $S$ and (b) $W$ parameter normalized to bulk values as a function of incident positron energy for as-grown, light-soaked, and 40-h annealed $a$-Si:H thin films. The bulk values $S_{\text {bulk }}$ and $W_{\text {bulk }}$ were measured in a separate measurement on a $c$-Si sample.

Fig. 1(b). The $W$ parameter for the as-grown $a$-Si:H is higher than that in $c$-Si, in agreement with the angular correlation data of Bhide et al. [23]. After light soaking the value of $W$ is very close to that for $c$-Si. It is this signature which we argue below to be due to defect dehydrogenation on light soaking.

The light soaking effect can more easily be seen in the $W / W_{\text {bulk }}$ vs $S / S_{\text {bulk }}$ plot shown in Fig. 2. This type of plot allows isolation of the different defect sites and assessment whether more than one defect type is involved, since each defect has its own $(S, W)$ coordinate [24]. Note that on the plot $c$-Si corresponds to the position $(1,1)$. For the as-grown film the data points lie along a straight line at all beam energies, indicating that the film has a homogeneous distribution of the same defect at all depths. The same is not true for the films after light soaking. Here one sees not 


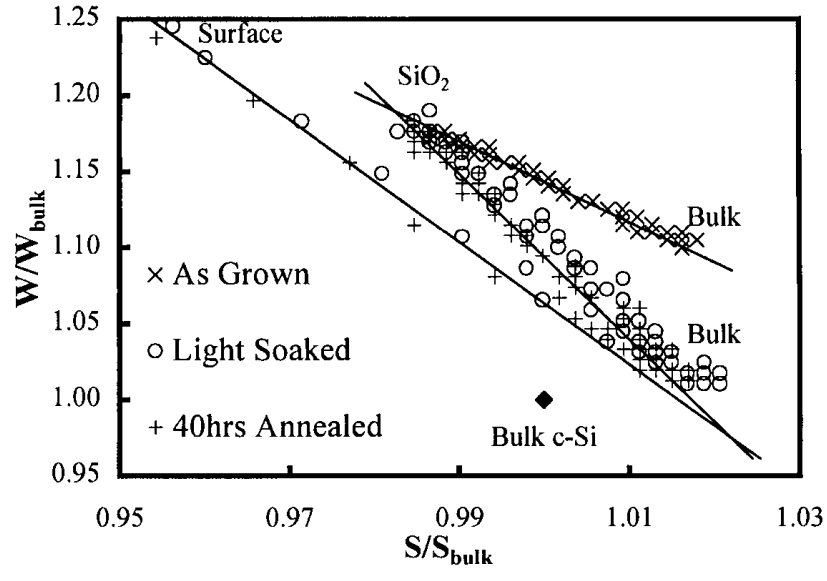

FIG. 2. The normalized core annihilation parameter $W$ versus the normalized valence annihilation parameter $S$, in as-grown, light-soaked, and 40-h annealed $a-\mathrm{Si}: \mathrm{H}$ samples. The regions corresponding to majority surface, bulk film, and quartz substrate annihilation are indicated, together with the data point corresponding to bulk crystalline silicon. The lines are guides to the eye.

only a different defect type in the bulk film on transferring from substrate to bulk, but in addition a very different surface and near surface defect type [characterized by low $S / S_{\text {bulk }}(\sim 0.96)$ and high $\left.W / W_{\text {bulk }}(\sim 1.25)\right]$ on transiting from bulk to surface. Some minor changes in defect signature occur on annealing in the surface region, which we suggest are due to hydrogen diffusion to the surface. More importantly, in the bulk region there is no reversal of the light soaking effect on thermal annealing, as would be expected if the changes in the line shape parameters were directly connected with the metastable defect sites responsible for the SWE.

Despite many PAS studies using both positron beams [21,22,25,26] and conventional sources [23,27-29], there is as yet no general consensus on the positron trapping sites in $a-\mathrm{Si}: \mathrm{H}$. Much of the uncertainty apparently arises from the variations in film preparation techniques $[21,25]$. However, evidence exists for four possible positron trapping states, namely, (i) coordination defects $(1 \nu)$ (ii) divacancylike defects $(2 \nu)$ (iii) vacancylike agglomerates comprising of $\sim 5$ missing atoms $(5 \nu)$, and (iv) large 10-20 diameter microvoids in which some positrons exist as bound $\mathrm{Ps}(\mathrm{MV})$. Both $5 \nu$ and $\mathrm{MV}$ states give rise to lifetime components in the range $400-550$ ps that are commonly seen in various $a-\mathrm{Si}: \mathrm{H}$ films [23,25-29]. The existence of microvoids is confirmed by the definite observation of positronium components in both lifetime and angular correlation studies [27,28].

The assertion that PECVD grown films contain $2 \nu$-type defects comes from positron lifetime studies [22,24], where the observed 310 to 350 ps component is identified with the $325 \mathrm{ps}$ lifetime of divacancies in $c$-Si [30]. A number of studies also find a short lifetime component (190-256 ps) from some films [23,27,28]. Such films presumably do not have sufficient divancy defects or agglomerates of higher volume present for saturation trapping, and so some positrons annihilate from either a delocalized Si-network state or from the site of saturated coordination defects that are present at the level of $10^{19} \mathrm{~cm}^{-3}$ in $a-\mathrm{Si}: \mathrm{H}$ [3]. The latter could be the shallow trapping sites of He et al. [27]. Since the mass of the hydrogen atom is small compared to the surrounding silicon atoms, $1 \nu$ - or $2 \nu$-type sites consisting of hydrogen saturated dangling bonds are expected to be able to act as positron traps.

The positron diffusion length obtained from the data in Fig. 1 is $L_{+}=100 \pm 30 \AA$, with little dependence on the light soaking condition. This is much shorter than the length typically observed in $c$-Si and is probably indicative as much of the greater degree of scattering experienced by diffusing positrons in an amorphous network compared to a crystalline lattice, as of a high density of trapping sites.

Although in the light-soaked state the $S$ parameter is seen to increase slightly, the dominant change is in the value of the $W$ parameter. The relative amount of core electron annihilations at a vacancy defect depends on the open volume of the defect and on the chemical nature of the surrounding atoms. On the other hand, mainly the valence electrons contribute to the value of the $S$ parameter, which thus depends predominantly on the open volume. We may conclude that while the open volume of the defect sites may increase slightly on light soaking, a change in the chemical nature of the open volume trapping sites causes fewer positron annihilations with core electrons. One of the most likely chemical environment changes is the removal of hydrogen from the defect structure. The presence of $\mathrm{H}$ saturating bonds within a defect could cause the positron to see higher momentum for two reasons. The first is that the Coulombic repulsion of the proton core of the hydrogen could have the effect of restricting the positron wave function, thus allowing more positron overlap with the $2 p$ core electrons of the $\mathrm{Si}$ atoms. The second is that the spatially confined $s p^{3}$ orbitals associated with the short $\mathrm{Si}-\mathrm{H}$ bond length $(\sim 1 \AA)$ would be expected to have high momentum components in the range of $20 \times 10^{-3} m_{0} c$. Thus the remarkable drop in the $W$ parameter on light soaking to close to a $c$-Si value can be explained simply on the basis that $\mathrm{Si}-\mathrm{H}$ bonds are being broken and $\mathrm{H}$ removed from the defect site.

We may now relate the above conclusion to the microscopic models of defect creation. The change in $W$ on light soaking is consistent with both the hydrogen emission mechanisms in (1) and (2). However, the change in $W$ is not seen to reverse on annealing. In the models the metastable ( $\mathrm{Si}-\mathrm{HH}-\mathrm{Si}$ ) complex is broken up on annealing, and the mobile hydrogen diffuses away. In the scheme of Branz [5] the mobile $\mathrm{H}$ can then be recaptured by the dangling bonds formed by the original emission process, i.e., reversing process (1). Godet in [6] does not treat the eventual fate of the mobile $\mathrm{H}$ released by annealing. However, it seems possible that the $\mathrm{Si}-\mathrm{Si}$ bonds formed by process 
(2) would have a low cross section for recapture of the mobile $\mathrm{H}$, i.e., that (2) is not reversed on annealing. The original doubly hydrogenated ( $\mathrm{Si}-\mathrm{HH}-\mathrm{Si}$ ) site is a likely positron trap, since the local density would be lower than other regions of the amorphous network. If the network adjustment on formation of the Si-Si bond of process (2) is not too great, the site will also be a positron trap after $\mathrm{H}$ emission. Thus our data tend to support the picture of [6], rather than [4] and [5], with positrons being captured and annihilating in the doubly hydrogenated ( $\mathrm{Si}-\mathrm{HH}-\mathrm{Si}$ ) sites to produce the large $W$ signature in the as-deposited material. Hydrogen is emitted from such sites on light soaking, causing the fall in $W$, but does not return on annealing.

In summary, we have shown how positron annihilation provides microscopic information on changes in open volume-type defects in undoped hydrogenated amorphous silicon before and after light soaking. The indication is that such defects are losing hydrogen on light illumination. This observation is consistent with the initiation of SWE and experiments that see a global expansion of the lattice on light soaking. Microscopic information gained from PAS places constraints on models of hydrogen motion fitting macroscopic Staebler-Wronski effect kinetics and may help to achieve a definitive description of metastability in $a-\mathrm{Si}: \mathrm{H}$.

[1] W. E. Spear and P. G. LeComber, Solid State Commun. 17, 1193 (1975).

[2] D. L. Staebler and C. R. Wronski, Appl. Phys. Lett. 31, 292 (1977).

[3] R. A. Street, Hydrogenated Amorphous Silicon (Cambridge University Press, Cambridge, England, 1991); also D. Redford and R.H. Bube, Photoinduced Defects in Semiconductors (Cambridge University Press, Cambridge, England, 1996), Chap. 4.

[4] R. Biswas and B. C. Pan, Appl. Phys. Lett. 72, 371 (1998).

[5] H. M. Branz, Solid State Commun. 105, 387 (1998).

[6] C. Godet, Philos. Mag. B 77, 765 (1998).

[7] R. Biswas, Qiming Li, B. C. Pan, and Y. Yoon, Phys. Rev. B 57, 2253 (1998).

[8] C. P. Herrero, Defect Diffus. Forum 153-155, 45 (1998).

[9] P. Hari, P. C. Taylor, and R. A. Street, in Amorphous Silicon Technology, MRS Symposia Proceedings No. A11.4 (Materials Research Society, Pittsburgh, 1994).

[10] N.H. Nickel and W.B. Jackson, J. Non-Cryst. Solids 164-166, 281 (1993).
[11] Denis P. Masson, Abdelhak Ouhlal, and Arthur Yelon, J. Non-Cryst. Solids 190, 151 (1995).

[12] Kong Guanglin, Zhang Dianlin, Zhao Yiping, and Liao Xianbo, in Solid State Phenomena (Scitec Publications, Zurich, Switzerland, 1995), Vols. 44-46, p. 677.

[13] T. Gotoh, S. Nonomura, S. Hirata, N. Masui, and S. Nitta, Sol. Energy Mater. Sol. Cells 49, 13 (1997).

[14] Zhao Yiping, Zhang Dianlin, Kong Guanglin, Pan Guangqin, and Liao Xianbo, Phys. Rev. Lett. 74, 558 (1995).

[15] Kong Guanglin, Zhang Dianlin, Yue Guozhen, and Liao Xianbo, Phys. Rev. Lett. 79, 4210 (1997).

[16] P. Hautojärvi and C. Corbel, in Positron Spectroscopy of Solids, edited by A. Dupasquier and A. P. Mills, Jr. (IOS Press, Amsterdam, 1995), p. 491.

[17] Xuanying Lin, Kuixun Lin, Yunpeng Yu, Y. W. Lam, Y. C. Chan, Shunhui Lin, and Florence Y. M. Chan, in Proceedings of First World Conference of Photovoltaic Energy Conversion, Waikoloa, Hawaii, 1994 (IEEE, New York, 1994), p. 462.

[18] C. D. Beling, S. Fung, H. M. Weng, C. V. Reddy, S. W. Fan, Y. Y. Shan, and C. C. Ling, in Slow Positron Beam Techniques for Solids and Surfaces, edited by Eric Ottewitte and Alex H. Weiss, AIP Conf. Proc. No. 303 (AIP, New York, 1994).

[19] A. Vehanen, K. Saarinen, P. Hautojärvi, and H. Huomo, Phys. Rev. B 35, 4606 (1987).

[20] Peter J. Schultz and K. G. Lynn, Rev. Mod. Phys. 60, 701 (1988).

[21] A. Van Veen, R. A. Hakvoort, H. Schut, and P.E. Mijnarends, J. Phys. IV (France) 5, C1-37 (1995).

[22] S. Muramatsu, R. Suzuki, L. Wei, and S. Tanigawa, Sol. Energy Mater. Sol. Cells 34, 525 (1994).

[23] V. G. Bhide, R. O. Dusdane, S. V. Rajarshi, A.D. Shaligram, and S. K. David, J. Appl. Phys. 62, 108 (1987).

[24] L. Liszkay, C. Corbel, L. Baroux, P. Hautojärvi, M. Bayhan, A. W. Brinkman, and S. Tatarenko, Appl. Phys. Lett. 64, 380 (1994).

[25] Y. Hiroyama, R. Suzuki, Y. Hirano, F. Sato, and T. Motooka, Jpn. J. Appl. Phys. 34, 5512 (1995).

[26] J. Störmer, P. Willutzki, D. T. Britton, G. Kögel, W. Triftshäuser, W. Kiunke, F. Wittmann, and I. Eisele, Appl. Phys. A 61, 71 (1995).

[27] Y. J. He, M. Hasegawa, R. Lee, S. Berko, D. Adler, and A. L. Jung, Phys. Rev. B 33, 5924 (1986).

[28] H.E. Schaefer, R. Würshum, R. Schwartz, D. Slobodin, and S. Wagner, Appl. Phys. A 40, 145 (1986).

[29] S. Dannefaer, D. Kerr, and B. G. Hogg, J. Appl. Phys. 54, 155 (1983).

[30] S. Dannefaer, G. W. Dean, D. P. Kerr, and B. G. Hogg, Phys. Rev. B 14, 2709 (1976). 